\title{
Emittance growth suppression with a multibunch feedback in high-energy hadron colliders: Numerical optimization of the gain and bandwidth
}

\author{
Sondre Vik Furuseth $\odot^{*}$ \\ CERN, 1211 Geneva, Switzerland, and EPFL, 1015 Lausanne, Switzerland \\ Xavier Buffat, Juan Sebastián Pereira-Cubillo®, and Daniel Valuch® \\ CERN, 1211 Geneva, Switzerland
}

(Received 10 September 2020; accepted 11 January 2021; published 27 January 2021)

\begin{abstract}
A transverse feedback system can effectively mitigate the emittance growth caused by injection oscillations and machine noise in hadron beams. However, as its action on the beam depends on beam position measurements of finite accuracy, it introduces additional noise on its own. The machine noise is in general strongest at low frequencies. Hence, the feedback is less needed at high frequencies. In this paper, two theories for the reduction of the machine noise induced emittance growth rate, with a bunch-by-bunch feedback, have been extended to a multibunch feedback. The extended theories show quantitative agreement with sophisticated macroparticle simulations. The emittance growth caused by the beam position measurement noise is numerically found to be only weakly dependent on the feedback's cutoff frequency, while it is strongly dependent on the single-bunch gain. The ultimate goal of this study is to find the optimal transverse feedback bandwidth and gain, determined by the minimization of the total emittance growth rate. The optimum depends on the ratio between the amplitudes of the beam position measurement error and the machine noise, the power spectrum of the machine noise, the response of the feedback filters, and the magnitude and details of the detuning. For the illustrative case of the Large Hadron Collier during collision in run 2, the optimum is found at the currently lowest possible cutoff frequency of $0.5 \mathrm{MHz}$, with a single-bunch damping time of approximately 270 turns. Using a chromaticity of 15 units, the minimal emittance growth rate at this cutoff frequency is $72 \%$ lower than with a bunch-by-bunch feedback. If the beam position measurement error can be reduced relative to the machine noise, the optimum will shift to larger single-bunch gains, or equivalently shorter single-bunch damping times.
\end{abstract}

DOI: 10.1103/PhysRevAccelBeams.24.011003

\section{INTRODUCTION}

The beams in synchrotrons perform many revolutions during their lifetimes. They also oscillate transversely around their ideal path with a finite amplitude because of injection errors, self-induced forces, such as wakefields, and various sources of external noise. The machine noise is typically strongest at low frequencies due to selfinductance in the electromagnets. In some machines, the beam screen also shields the beams from high frequency field variations [1]. That is, in general, also true for other sources of external noise, such as the ground motion [2]. From here on, all of these forces are referred to as the machine noise. In the Large Hadron Collider (LHC),

\footnotetext{
*sondre.vik.furuseth@cern.ch
}

Published by the American Physical Society under the terms of the Creative Commons Attribution 4.0 International license. Further distribution of this work must maintain attribution to the author(s) and the published article's title, journal citation, and DOI. machine noise may be dominated by fluctuations in power converters, which are currently understood to be strongest at frequencies below $10 \mathrm{kHz}$ [3]. Such a low-frequent (LF) machine noise will kick all particles in a bunch equally, both those in the head and in the tail. Furthermore, such an LF machine noise can only excite multibunch modes of equally low frequencies.

Transverse beam oscillations can both lead to emittance growth and self-amplified instabilities, reducing the beam quality in either case. To minimize these oscillations, it is common to act on the beams with transverse bunch feedback systems [4,5], sometimes referred to as dampers, reducing the transverse momentum of a bunch by a gain every turn. Transverse feedbacks can be used to minimize rigid-bunch oscillations, but also intrabunch head-tail motion [6]. The use and capabilities of the feedback depend on the beam requirements.

Since the beams can have speeds close to the speed of light, one cannot act on a bunch at the same turn as the measurement of the transverse displacement. This requires a prediction of where the bunch will be at a later turn, which is 
based on the assumed tune. If the prediction is sufficiently wrong, and/or the feedback gain is too large, the feedback system may destabilize the beams [7]. The tune acceptance will not be studied in this paper. A key parameter when considering the stability of the feedback loop is the group delay of the feedback filter [8]. Much work has been put into designing optimal multiturn filters, damping a bunch based on the measurements from more than one turn. Different filters have different stability limits [9].

The transverse oscillations are measured by a beam position monitor (BPM), often using electromagnetic pickups that have finite accuracy and precision. The error between the measured position and the actual position of a bunch will from here on be referred to as the BPM error. This error consists both of a systematic and a stochastic part. The systematic BPM error depends on the beam position in the pickup. It is not relevant within the scope of this paper because the turn-by-turn measurements are notch-filtered to suppress the closed orbit and thereby also the systematic error. The main sources of the stochastic BPM error in the LHC are the pickup measurement noise and the intrinsic noise of the analog-to-digital conversion process. The quantization noise due to the digitization of the signal is negligible in comparison.

The BPM error is fed back into the beam by the feedback and causes additional degradation of the beam quality. The unwanted kicks from the feedback will be referred to as the BPM noise. It has been postulated that the impact of the BPM noise may be reduced by damping a bunch based on the measurements of both itself and its closest neighbors, corresponding effectively to operating the feedback in a lowbandwidth (LBW) setting with an upper cutoff frequency $f_{\text {cutoff }}$ smaller than the bunch repetition frequency. An LBW feedback should also be more efficient at damping the multibunch modes driven by LF machine noise.

An optimal feedback minimizes the emittance growth rate, driven by the BPM noise and the machine noise, and maintains beam stability. In this paper, we introduce a model of an LBW feedback and search for the optimal cutoff frequency and gain. The optimum will depend on the machine noise, the BPM noise, the feedback filter, and the feedback stability limit.

Note that there are two main sources of emittance growth in a hadron beam [10]: (i) Single-particle kicks, e.g., caused by intrabeam scattering; (ii) Dipolar kicks, affecting all particles in the same longitudinal slice equally. If one neglects stochastic cooling, a feedback can only reduce the emittance growth caused by the dipolar kicks.

\section{THEORY}

\section{A. Emittance growth with ideal bunch-by-bunch feedback}

An ideal bunch-by-bunch feedback works by measuring the transverse position of bunch $b$ at either multiple locations or multiple turns, to have an accurate estimate of its normalized phase space coordinates $\left(x_{b}, p_{b}\right)$ at the location of the feedback actuator, where the momentum is reduced by a gain $g$ as

$$
p_{b} \rightarrow p_{b}-g p_{b}=p_{b}(1-g) .
$$

The feedback kick has to be performed at a later turn due to special relativity. This feedback corresponds to a damping time of $\tau_{g}=2 / g$, in number of turns.

The expressions for the suppression of the emittance growth rate with an ideal bunch-by-bunch feedback that will be introduced in this section, were originally derived under certain assumptions on the noise. First of all, the noise was considered to affect all particles in a bunch equally. This requires the noise to be negligible at frequencies similar to or higher than the inverse bunch length. This requirement is met in the LHC, where the noise is negligible at frequencies $\gtrsim 1 \mathrm{GHz}$ [4]. Secondly, the power spectral density of the noise is assumed to be flat over the bunch spectrum. The width of the bunch spectrum is typically small, often making this a valid assumption. On the other hand, if there are narrow spectral lines in the noise spectrum that overlap with the bunch spectrum, that is not covered by this theory. This can occur, if care is not taken, due to the $50 \mathrm{~Hz}$ lines observed in the LHC [3]. Finally, the derivation assumes small noise kicks, keeping only the lowest-order terms. If these assumptions are met, what matters is the power spectral density of the noise at the bunch's betatron frequency and corresponding alias frequencies. The exact histogram of the small kicks, be it, e.g., Gaussian or a box function, does not matter. In this section, the noise is represented by a flat power spectrum up to a half-integer multiple of the revolution frequency $f_{\text {rev }}$, with a variance per single kick per turn of $\sigma_{\text {Noise }}^{2}$, representing the quadratic sum of the machine noise amplitude normalized to the beam divergence at the location of the noise sources.

If the decoherence is caused by incoherent detuning, as that created by Landau octupoles, the emittance growth rate per turn in either transverse plane is given by [11]

$$
\frac{\dot{\varepsilon}}{\varepsilon_{0}}=\frac{\sigma_{\text {Noise }}^{2}}{2}\left\langle\frac{\left(1-\frac{g}{2}\right)^{2} 4 \pi^{2} \Delta Q^{2}}{\left(\frac{g}{2}\right)^{2}+\left(1-\frac{g}{2}\right) 4 \pi^{2} \Delta Q^{2}}\right\rangle,
$$

where $\varepsilon_{0}$ is the initial emittance, the angle brackets signify an average over the bunch distribution, and $\Delta Q$ is the detuning of a single particle relative to the average tune in the considered plane of all the particles in the bunch, not relative to the bare machine tune. The horizontal detuning due to Landau octupoles [12], relative to the average tune of all the particles, can be given as

$$
\Delta Q_{x}=a_{x}\left(J_{x}-\left\langle J_{x}\right\rangle\right)+b_{x}\left(J_{y}-\left\langle J_{y}\right\rangle\right),
$$


where $a_{x}$ and $b_{x}$ are the in-plane and cross-plane detuning coefficients, respectively, later assumed to be equal in both planes, and $J_{j}$ is the normalized action in plane $j \in\{x, y\}$. The root-mean-square (rms) tune spread is $\sqrt{a_{x}^{2}+b_{x}^{2}}$, assuming a Gaussian bunch distribution.

If the detuning is driven by a single coherent head-on beam-beam interaction per turn, in a configuration with round and symmetric beams, the emittance growth rate per turn without chromaticity is given by [13]

$$
\frac{\dot{\varepsilon}}{\varepsilon_{0}} \approx \frac{1-s_{0}}{2} \frac{\sigma_{\text {Noise }}^{2}}{2}\left(1+\frac{g}{2 \pi \xi_{\text {tot }}}\right)^{-2},
$$

where $s_{0} \approx 0.645$ is a constant determined numerically for the case of round beams, and $\xi_{\text {tot }}$ is the total beam-beam parameter that characterizes the maximum tune shift of the particles in the center of the bunch. For round beams, the beam-beam parameter of one head-on interaction is given by [13]

$$
\xi=\frac{n_{P} r_{P}}{4 \pi \varepsilon_{n}},
$$

where $n_{P}$ is the number of protons per bunch, $r_{P}$ is the classical proton radius, and $\varepsilon_{n}$ is the normalized transverse emittance, which is assumed equal in both planes. The rms tune spread is approximately $0.168 \xi_{\text {tot }}$ [14]. Since the incoherent tune spectrum due to beam-beam interactions does not contain the coherent $\sigma$ - and $\pi$ - beam-beam modes, the beam-beam driven decoherence is less effective than that of incoherent detuning sources. As a result, shown in more detail in Sec. III, the emittance growth rate is smaller with a coherent beam-beam interaction than with octupole detuning of the same rms tune spread.

\section{B. Real LBW feedback}

Due to the finite accuracy of the BPMs, the measured positions are not exact, leading to an error between the predicted momentum $p_{b p}$ at the feedback actuator and the actual momentum $p_{b}$. Therefore, the effect of the ideal bunch-by-bunch feedback in Eq. (1) changes to

$$
p_{b} \rightarrow p_{b}-g p_{b p}=p_{b}(1-g)+g \delta_{b p},
$$

where $g \delta_{b p}$ is the BPM noise kick. The stochastic BPM error will be modeled as drawn from a centered normal distribution, $\delta_{b p} \sim \mathcal{N}\left(0, \sigma_{\mathrm{BPM}}^{2}\right)$. This assumption is not essential to the key results. The possible additional error due to the prediction is neglected. The emittance growth rate caused by the BPM noise can be found by exchanging $\sigma_{\text {Noise }}$ in Eqs. (2) and (4) by $g \sigma_{\mathrm{BPM}}$. It cannot be reduced by increasing the feedback gain.

A multibunch/LBW feedback works by acting on multiple bunches based on the measurement of one bunch, or equivalently acting on one bunch $b$ based on the measurements of multiple bunches $b^{\prime}$. This can be written

$$
p_{b} \rightarrow p_{b}-g \sum_{b^{\prime}} \epsilon_{b^{\prime}} w_{b b^{\prime}} p_{b^{\prime} p}
$$

where the coefficient

$$
\epsilon_{b^{\prime}}= \begin{cases}1, & \text { bunch } b^{\prime} \text { exists } \\ 0, & \text { otherwise }\end{cases}
$$

marks the bunches and $w_{b b^{\prime}}$ is the response function of the complete transverse feedback filter, from the position measurement to the feedback kick experienced by the bunches, normalized so that $w_{b b}=1$. The complete transverse feedback filter consists of the combined response due to several individual digital filters, the high power tube amplifiers, and the kicker parameters in the signal processing chain.

Response functions for two types of low-pass feedback systems, with various cutoff frequencies $f_{\text {cutoff }}$, are visualized in Fig. 1(a). The corresponding transfer functions are displayed in Fig. 1(b). The extended bandwidth (Ext. BW) is for all intents and purposes a bunch-by-bunch feedback. The response function for the exponential filter ("EXP") is the exponential function,

$$
w_{b b^{\prime}}^{\mathrm{EXP}}=\exp \left(-2 \pi f_{\text {cutoff }} \Delta t\left|b-b^{\prime}\right|\right),
$$

where $\Delta t$ is the bunch separation. The response functions labeled "ADT" include all the filtering in the digital signal processing and the analogue chain frequency response of the LHC transverse feedback, assuming known bunch measurements [5]. In addition to the measured center bunch, the response extends symmetrically over the 32 closest bunch slots on either side of this bunch. The lowest reasonable cutoff frequency for the LHC transverse feedback is $0.5 \mathrm{MHz}$. However, this can be reduced further if found necessary. The additional high-pass filter required to make a bandpass filter is not relevant in this paper.

The LBW feedback is most valuable when the beam tends to oscillate with low-frequency modes. To appreciate the effect of an LBW feedback, the transverse beam oscillation at the BPM can be decomposed into the sum of a multibunch mode $p_{b m}=A_{m} \cos \left(2 \pi f_{m} t_{b}+\phi_{0 m}\right) \equiv A_{m} \cos \left(\phi_{b m}\right)$, denoted by the subscripted $m$, and an individual momentum $p_{b i} \sim \mathcal{N}\left(0, \sigma_{i}^{2}\right)$ per bunch. By inserting this decomposition and expanding the cosine in $p_{b m}$, Eq. (7) can be rewritten as

$$
\begin{aligned}
p_{b} & \rightarrow p_{b}-g \sum_{b^{\prime}} \epsilon_{b^{\prime}} w_{b b^{\prime}}\left(p_{b^{\prime} m}+p_{b^{\prime} i}-\delta_{b^{\prime} p}\right) \\
= & p_{b m}\left(1-g_{b m}\right)+p_{b i}(1-g)+g \delta_{b \Sigma i}+g \delta_{b \Sigma p} \\
& +g A_{m} \sin \left(\phi_{b m}\right) \sum_{b^{\prime}} \epsilon_{b^{\prime}} w_{b b^{\prime}} \sin \left[2 \pi f_{m} \Delta t\left(b^{\prime}-b\right)\right],
\end{aligned}
$$

where 


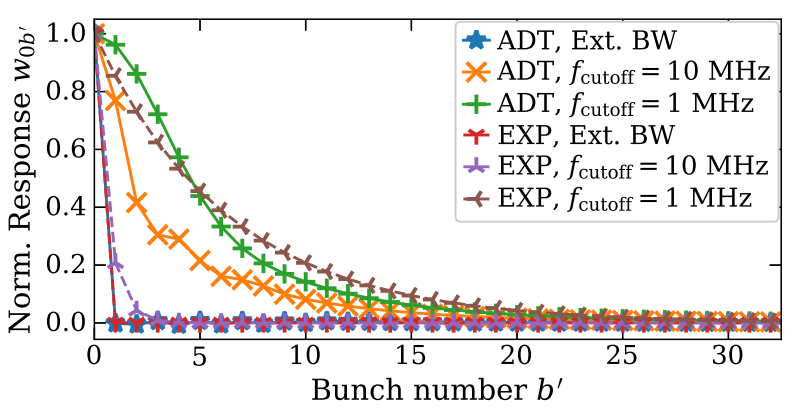

(a)

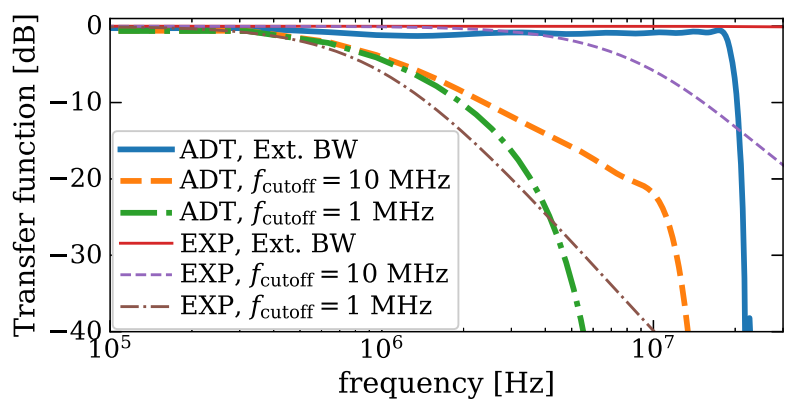

(b)

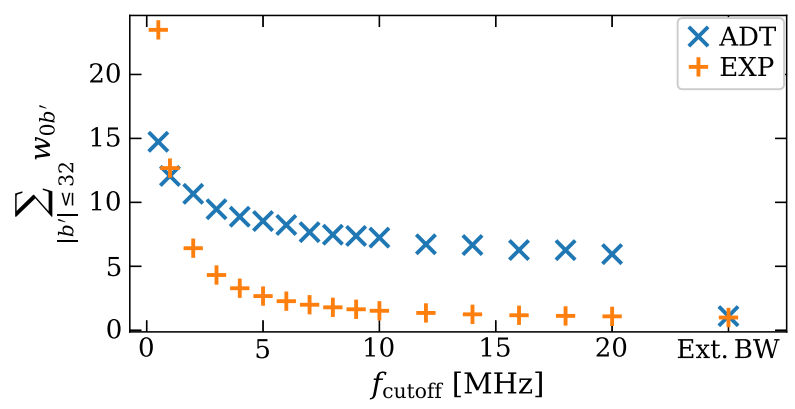

(c)

FIG. 1. Response functions of two types of filters in time domain in (a) and in frequency domain in (b), assuming a bunch separation of $\Delta t=25 \mathrm{~ns}$. The sums of the response functions in time domain, over all neighbors maximally 32 slots away, are given in (c). The EXP filter is an exponential filter, while the ADT filter replicates the transverse feedback in the LHC, both of which are fully symmetric in time in the range $\left|b-b^{\prime}\right| \leq 32$, although only $b^{\prime}-b \geq 0$ is shown here.

$$
\begin{gathered}
g_{b m}=g \sum_{b^{\prime}} \epsilon_{b^{\prime}} w_{b b^{\prime}} \cos \left[2 \pi f_{m} \Delta t\left(b^{\prime}-b\right)\right] \leq g \tilde{N}, \\
\delta_{b \Sigma i}=-\sum_{b^{\prime} \neq b} \epsilon_{b^{\prime}} w_{b b^{\prime}} p_{b^{\prime} i} \sim \mathcal{N}\left(0,\left(\tilde{N}_{b}-1\right) \sigma_{i}^{2}\right), \\
\delta_{b \Sigma p}=\sum_{b^{\prime}} \epsilon_{b^{\prime}} w_{b b^{\prime}} \delta_{b^{\prime} p} \sim \mathcal{N}\left(0, \tilde{N}_{b} \sigma_{B P M}^{2}\right), \\
\tilde{N}_{b}=\sum_{b^{\prime}} \epsilon_{b^{\prime}} w_{b b^{\prime}},
\end{gathered}
$$

The individual single-bunch motion will be damped with the single-bunch gain $g$ as before. The multibunch motion will be damped with an effective multibunch gain $g_{b m}$. The individual motion results in a kick $g \delta_{b \Sigma i}$ per turn and the BPM errors result in a kick $g \delta_{b \Sigma p}$ per turn, both acting as a noise with the same spectrum as the feedback. The last term in Eq. (10) originates in the multibunch motion of the beam, $p_{b m}$. It can lead to an emittance growth if not suppressed. It can be minimized by: (i) reducing $g$; (ii) reducing $A_{m}$, which requires a large $g_{b m}$; (iii) reducing the summands, which requires $f_{m} \ll f_{\text {cutoff }}$, which is also required to get a large $g_{b m}$; (iv) using a symmetric filter so that the summands cancel. If the bunches are gathered in trains of consecutively filled bunch slots, separated by multiple empty slots, for which $\epsilon_{b^{\prime}}=0$ [15], the last alternative does not work for the bunches at either end of a train.

If the cutoff frequency is larger than the multibunch mode frequency, $f_{\text {cutoff }} \gg f_{m}$, the multibunch gain will be larger than the single-bunch gain, $g_{b m}=g \tilde{N}_{b} \geq g$. The sum of the peak-normalized response function is illustrated for various cutoff frequencies with both considered filter types in Fig. 1(c). Since this is the maximum ratio between the multibunch gain and the single-bunch gain, $\max \left(g_{b m} / g\right)$, it can be seen from this figure that reducing from the Ext. BW to standard operation of the ADT with $f_{\text {cutoff }}=20 \mathrm{MHz}$ may already lead to a significant increase of the effective multibunch gain. The same change of bandwidth will only have a marginal impact when using the EXP filter.

\section{Emittance growth with LBW feedback}

The motion of the beam can more accurately be modeled as a sum of multibunch modes. For a revolution frequency $f_{\text {rev }}$, the horizontal multibunch modes have frequencies

$$
f_{m}=f_{\text {rev }} \cdot\left|m+Q_{x}\right|, \quad m \in \mathbb{Z},
$$

where $Q_{x}$ is the fractional horizontal tune. Due to aliasing, the only modes of interest are those with frequencies $f_{m} \in\left[0, f_{\mathrm{rf}} / 2\right]$, where $f_{\mathrm{rf}}=M f_{\mathrm{rev}}$ is the bunch slot frequency, and $M$ is the number of equidistant bunch slots.

Noise at a certain frequency $f \in\left[0, f_{\mathrm{rf}} / 2\right]$, will only excite multibunch motion at that frequency. The reduction of the emittance growth rate with incoherent detuning in Eq. (2) becomes a sum over the multibunch modes

$$
\frac{\dot{\varepsilon}_{b}}{\varepsilon_{0}}=\sum_{m} \frac{S_{\mathrm{Noise}}\left(f_{m}\right)}{2}\left\langle\frac{\left(1-\frac{g_{b m}}{2}\right)^{2} 4 \pi^{2} \Delta Q^{2}}{\left(\frac{g_{b m}}{2}\right)^{2}+\left(1-\frac{g_{b m}}{2}\right) 4 \pi^{2} \Delta Q^{2}}\right\rangle .
$$


Equivalently, one gets a new expression for the reduction of the emittance growth rate with a beam-beam interaction given by Eq. (4) as

$$
\frac{\dot{\varepsilon}_{b}}{\varepsilon_{0}} \approx \frac{1-s_{0}}{2} \sum_{m} \frac{S_{\text {Noise }}\left(f_{m}\right)}{2}\left(1+\frac{g_{b m}}{2 \pi \xi_{\text {tot }}}\right)^{-2} .
$$

Here, $g_{b m}$ is the effective gain in Eq. (11), which depends on $f_{m}$ and the distribution of neighbors, and $S_{\text {Noise }}$ is the power spectral density of the noise.

If the noise spectrum is flat up to an upper frequency $f_{\max }$, the power spectral density of the noise is, by the Plancherel theorem [16] and our convention of normalization, related to the noise spread in time as

$$
S_{\text {Noise }}(f)= \begin{cases}\sigma_{\text {Noise }}^{2} \frac{f_{\text {rev }}}{2 f_{\text {max }}}, & f \in\left[0, f_{\text {max }}\right] \\ 0, & \text { otherwise }\end{cases}
$$

In the case of a single bunch $(M=1)$ affected by white noise, the effective noise has $f_{\max }=f_{\text {rev }} / 2$, since the bunch only samples the noise once per turn. As a consequence, one gets $S_{\text {Noise }}\left(f \leq f_{\max }\right)=\sigma_{\text {Noise }}^{2}$. In the case of $M$ evenly spaced bunch slots and a flat noise spectrum up to $f_{\max }=f_{\text {rf }} / 2$, one gets $S_{\text {Noise }}(f)=\sigma_{\text {Noise }}^{2} / M$. For an ideal Ext. BW feedback, $g_{b m}=g$, Eqs. (17) and (18) are reduced to Eqs. (2) and (4), respectively, as the sum will be over $M$ multibunch modes. Note that only the machine noise is considered in Eq. (19). The BPM noise will be filtered by the LBW filter, and cannot therefore be included in this manner.

\section{RESULTS}

Simulations have been run with the tracking code COMBI [17-19] to study the emittance growth rate driven by both LF machine noise and BPM noise, while being damped by various LBW feedbacks. The standard numerical setup is summarized in Table I. Variations to this setup are addressed in the text. All bunches in a beam are grouped together in a single train, filling consecutive bunch slots. The tune spread is caused by Landau octupoles, given by Eq. (3), unless stated otherwise. The prediction of the momentum after the feedback group delay always assumes the bare machine tunes $(0.31,0.32)$. The LF machine noise used has a flat power spectrum up to $f_{\max }$, being an integer multiple of $f_{\text {rev }}$. The noise signal has been generated by filtering the Fourier transform of finite length signals of Gaussian white noise, whereupon the signals have been concatenated in such a way as to erase the transition [20,21]. The noise is typically strongest at the lowest betatron sideband frequency, which varies between machines. The specific choice of $f_{\text {rev }}$ does not have a direct impact on the results. The BPM error is added to the phase space measurement before being used to calculate the response on the various bunches. The emittance growth rate
TABLE I. Numerical parameters used in the simulations.

\begin{tabular}{lcc}
\hline \hline Parameter & Unit & Value \\
\hline Bunches per beam & {$[1]$} & 128 \\
Macroparticles per bunch & {$[\mathrm{p} / \mathrm{b}]$} & $10^{6}$ \\
Number of turns & {$[1]$} & $10^{5}$ \\
Bunch spacing, $\Delta t$ & {$[\mathrm{~ns}]$} & 25 \\
Revolution frequency, $f_{\text {rev }}$ & {$[\mathrm{kHz}]$} & 11.245 \\
Fractional horizontal tune, $Q_{x}$ & {$[\mathrm{mod} \mathrm{1]}$} & 0.31 \\
Fractional vertical tune, $Q_{y}$ & {$[\mathrm{mod} \mathrm{1}]$} & 0.32 \\
Synchrotron tune, $Q_{s}$ & {$\left[10^{-3}\right]$} & 1.9 \\
RMS momentum spread, $\sigma_{\delta}$ & {$[1]$} & $10^{-4}$ \\
Linear chromaticity, $Q^{\prime}$ & {$[1]$} & 0 \\
Octupole in-plane coeff., $a_{x}{ }^{\mathrm{a}}$ & {$\left[10^{-3}\right]$} & 1.09 \\
Octupole cross-plane coeff., $b_{x}{ }^{\mathrm{a}}$ & {$\left[10^{-3}\right]$} & -0.761 \\
Beam beam parameter, $\xi_{\text {tot }}$ & {$\left[10^{-3}\right]$} & 7.9 \\
Single-bunch gain, $g$ & {$[1 / \mathrm{turn}]$} & 0.01 \\
Feedback group delay & {$[$ turn $]$} & 4 \\
Machine noise amplitude, $\sigma_{\text {Noise }}{ }^{\mathrm{b}}$ & {$\left[\sigma_{p}\right.$ turn $\left.{ }^{-\frac{1}{2}}\right]$} & $10^{-3}$ \\
Upper noise frequency, $f_{\text {max }}$ & {$\left[f_{\text {rev }}\right]$} & 1 \\
\hline
\end{tabular}

${ }^{a}$ Equal also in the vertical plane.

${ }^{\mathrm{b}}$ Noise given in units of the rms beam divergence.

is presented in units of a reference emittance growth rate. Unless stated otherwise, this reference is the emittance growth rate per turn expected with full decoherence,

$$
\frac{\dot{\varepsilon}_{\text {ref }}}{\varepsilon_{0}}=\frac{\sigma_{\text {Noise }}^{2}}{2} .
$$

\section{A. Dependence on feedback filter, cutoff frequency, noise type, and source of detuning}

The emittance growth rate of all 128 bunches, occupying consecutive bunch slots in one train, that are kicked by an LF noise and damped with a single-bunch gain $g=0.01$, is presented in Fig. 2. The agreement with the theory in Eq. (17) is equally good for the Ext. BW feedback as after the extension of the theory to an LBW feedback. The bunches at either end of the train do not benefit maximally from the LBW configuration, due to the lack of neighbors, and thus have a smaller effective multibunch gain. Note that the ADT filter improves significantly more than the EXP filter when going from Ext. BW to $f_{\text {cutoff }}=20 \mathrm{MHz}$. This result was expected based on the sums of the normalized response functions displayed in Fig. 1(c).

The emittance growth rate has been calculated with $f_{\text {cutoff }} \in\{0.5,1,2,5,10,20\} \mathrm{MHz}$, in addition to the Ext. BW. The average emittance growth rate of all 128 bunches, kicked only by either LF noise or BPM noise, is displayed as a function of $f_{\text {cutoff }}$ in Fig. 3. For the cases with only BPM noise, the reference emittance growth rate per turn is $\dot{\varepsilon}_{\text {ref }} / \varepsilon_{0}=\left(g \sigma_{\mathrm{BPM}}\right)^{2} / 2$. The emittance growth rate driven by LF noise is reduced significantly by reducing $f_{\text {cutoff }}$, and the different dependence on $f_{\text {cutoff }}$ for the two filter types is unmistakable. The emittance growth rate driven by BPM 


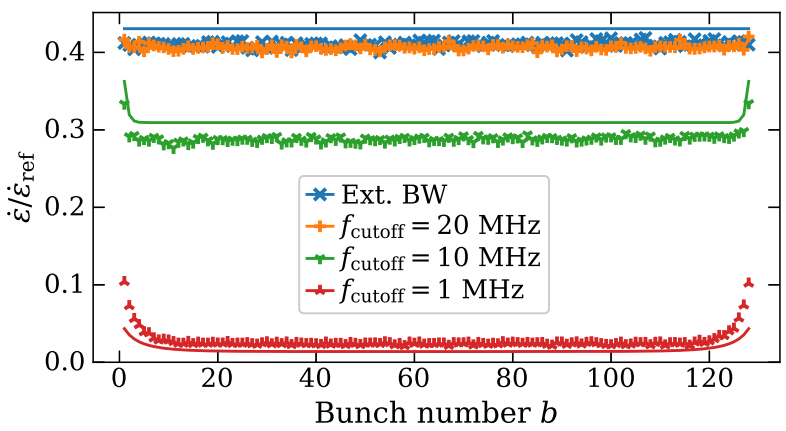

(a)

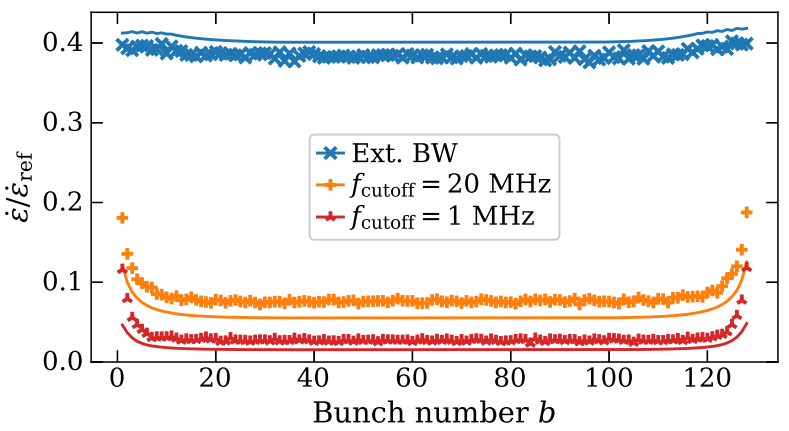

(b)

FIG. 2. Emittance growth rate for all 128 neighboring bunches affected by only LF noise, using the EXP filter in (a) and the ADT filter in (b). The solid lines are the expected emittance growth rates, calculated with Eqs. (11) and (17).

noise also depends on $f_{\text {cutoff }}$, but to a lesser extent. The main approach to reduce BPM noise driven emittance growth is to reduce the single-bunch gain.

The average emittance growth rate with LF noise, of various maximum frequencies $f_{\max }$, is displayed in Fig. 4 . As long as $f_{\max } \leq 1 \mathrm{MHz}$, there is only a marginal increase in the emittance growth rate for the tested values of $f_{\text {cutoff }}$. As a rule of thumb, reducing the cutoff frequency of the feedback only reduces the emittance growth rate as long as

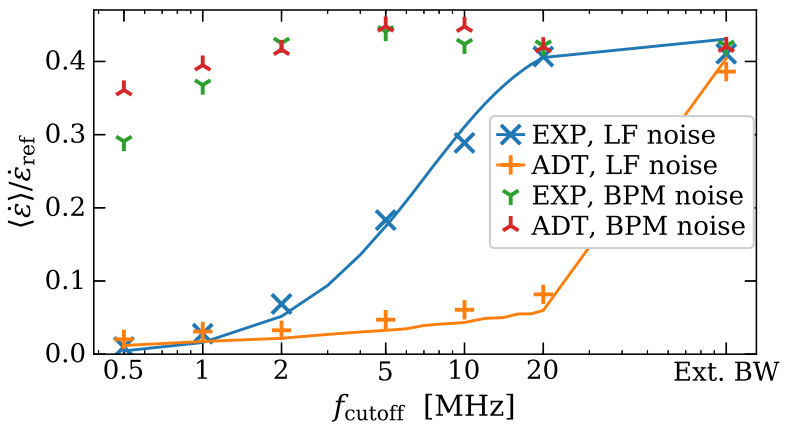

FIG. 3. Average emittance growth for 128 neighboring bunches, affected by either LF noise or BPM noise, using the EXP filter and the ADT filter. The solid lines are the expected emittance growth rates due to LF noise, calculated with Eqs. (11) and (17).

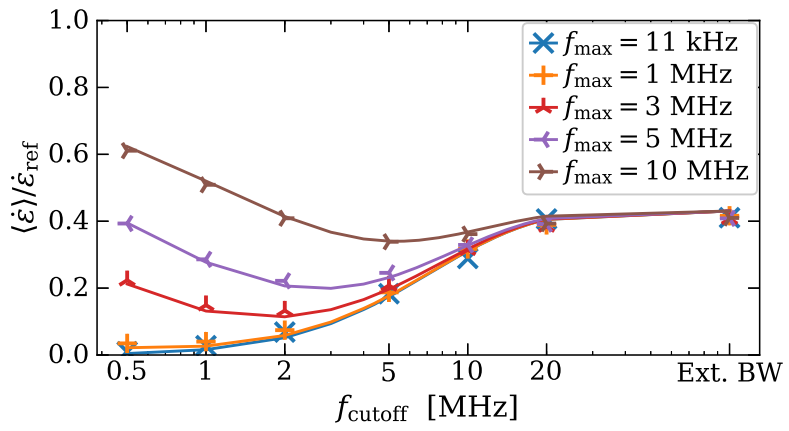

(a)

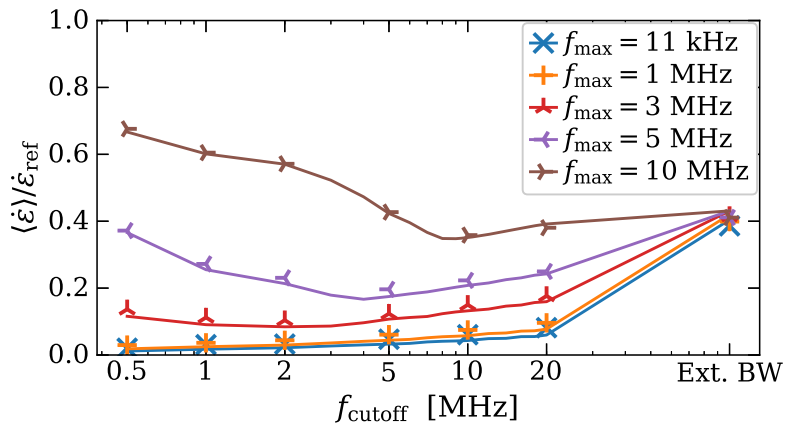

(b)

FIG. 4. Average emittance growth for 128 neighboring bunches affected by LF noise of various $f_{\text {max }}$, using the EXP filter in (a) and the ADT filter in (b). The solid lines are the expected emittance growth rates, calculated with Eqs. (11) and (17).

it still covers the power spectrum of the noise. Note that the power spectrum is flat for all values of $f_{\max }$ in these simulations. The noise in a machine will in general be weaker at higher frequencies.

The average emittance growth with a beam-beam interaction, in comparison to octupole detuning, is displayed in Fig. 5. The agreement with the theory for a beam-beam interaction in Eq. (18) is equally good as the theory for octupole detuning in Eq. (17). The emittance growth rate with a beam-beam interaction and zero chromaticity $Q^{\prime}=0$ is significantly lower than with octupole detuning. However, with the introduction of chromaticity, the emittance growth rate increases substantially. In comparison, the emittance growth rate increases more due to the introduction of chromaticity in addition to a beam-beam interaction, than in addition to octupole detuning. This is due to a shift of the coherent beam-beam modes due to chromaticity, as was discussed in greater detail in [22].

\section{B. Optimal feedback in the LHC}

The goal of this section is to find the optimal cutoff frequency and gain for the LHC in collision, both now and in the future. In the LHC as of 2018, the ratio between the BPM error and LF noise was in both planes of both beams $\sigma_{\mathrm{BPM}} / \sigma_{\mathrm{Noise}} \approx 50$ [22]. In this section, the emittance growth rate will be presented in units of $\dot{\varepsilon}_{\text {ref }} / \varepsilon_{0}=\sigma_{\text {Noise }}^{2} / 2$, the 


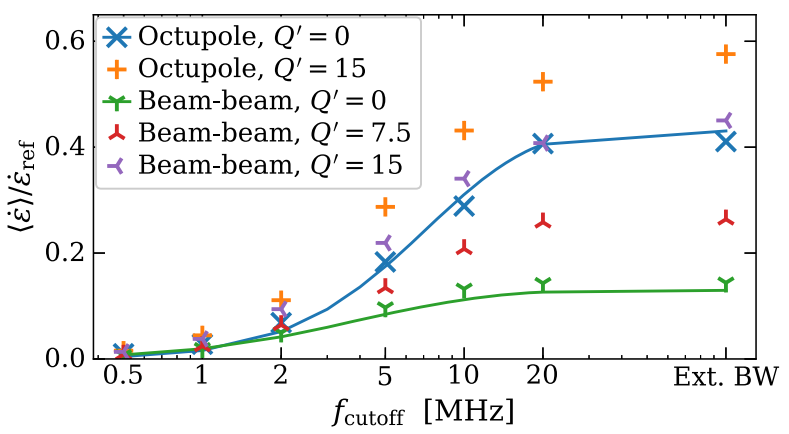

(a)

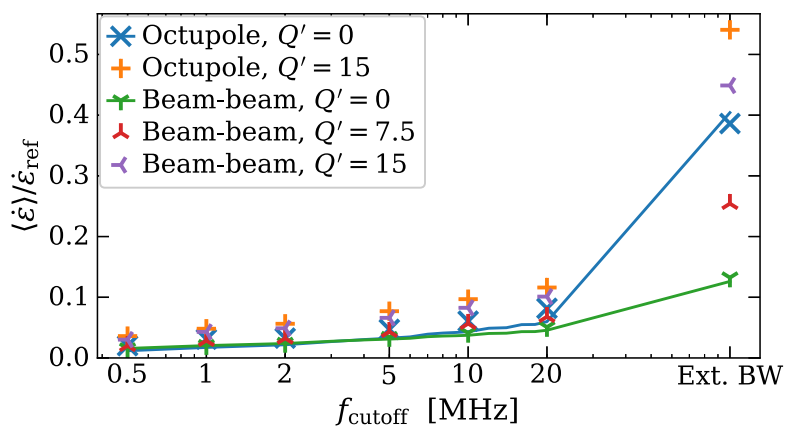

(b)

FIG. 5. Average emittance growth for 128 neighboring bunches affected by LF noise, using the EXP filter in (a) and the ADT filter in (b). The solid lines are the expected emittance growth rates, calculated with Eq. (11) and either Eq. (17) for octupole detuning or Eq. (18) for beam-beam interactions.

relative rate expected due to the $\mathrm{LF}$ noise with full decoherence, also for the simulations with only BPM noise. The source of detuning will always be beam-beam interactions, as we want to minimize the emittance growth rate in the LHC in collision. For the same reason, all simulations are run with the ADT filter. Note that we use a simplified model of the LHC with a single head-on interaction per turn, not multiple as was studied for the bunch-by-bunch feedback in [22], and not including long-range interactions. This is an acceptable choice in this paper, since the main focus is the impact of the feedback bandwidth on the emittance growth rate, and the main impact of the beam-beam interactions on the feedback efficiency is through the rms tune spread. Until further notice, the total beam-beam parameter remains at $\xi_{\text {tot }}=0.0079$.

The simulated average emittance growth rate of 128 bunches, with $Q^{\prime}=0$ and either only LF noise or BPM noise or both, is presented as a function of both the feedback's cutoff frequency and the single-bunch gain in Fig. 6. The emittance growth rate caused by the LF noise is as expected reduced by either increasing $g$ or reducing $f_{\text {cutoff }}$. The emittance growth rate caused by the BPM noise is strongly dependent on $g$. The emittance growth rates in the simulations with both types of noise are equal to the sum of the growth rates found with each type of noise
TABLE II. Minimal emittance growth rate and corresponding optimal gain for a given cutoff frequency, beam-beam parameter, noise ratio, and chromaticity.

\begin{tabular}{|c|c|c|c|c|}
\hline \multirow[b]{3}{*}{$f_{\text {cutoff }}$} & \multicolumn{4}{|c|}{$\xi_{\text {tot }}=7.9 \times 10^{-3}, \sigma_{\mathrm{BPM}}=50 \sigma_{\text {Noise }}$} \\
\hline & \multicolumn{2}{|c|}{$Q^{\prime}=0$} & \multicolumn{2}{|c|}{$Q^{\prime}=15$} \\
\hline & $g\left[10^{-2}\right]$ & $\dot{\varepsilon}\left[\dot{\varepsilon}_{\mathrm{ref}}\right]$ & $g\left[10^{-2}\right]$ & $\dot{\varepsilon}\left[\dot{\varepsilon}_{\mathrm{ref}}\right]$ \\
\hline Ext. BW & 0.67 & 0.165 & 1.41 & 0.523 \\
\hline $20 \mathrm{MHz}$ & 0.92 & 0.095 & 0.94 & 0.225 \\
\hline $0.5 \mathrm{MHz}$ & 0.70 & 0.070 & 0.75 & 0.149 \\
\hline
\end{tabular}

\begin{tabular}{|c|c|c|c|c|}
\hline \multirow[b]{3}{*}{$f_{\text {cutoff }}$} & \multicolumn{4}{|c|}{$\xi_{\text {tot }}=7.9 \times 10^{-3}, \sigma_{\mathrm{BPM}}=25 \sigma_{\text {Noise }}$} \\
\hline & \multicolumn{2}{|c|}{$Q^{\prime}=0$} & \multicolumn{2}{|c|}{$Q^{\prime}=15$} \\
\hline & $g\left[10^{-2}\right]$ & $\dot{\varepsilon}\left[\dot{\varepsilon}_{\text {ref }}\right]$ & $g\left[10^{-2}\right]$ & $\dot{\varepsilon}\left[\dot{\varepsilon}_{\text {ref }}\right]$ \\
\hline Ext. BW & 2.83 & 0.130 & 4.58 & 0.293 \\
\hline $20 \mathrm{MHz}$ & 2.07 & 0.049 & 2.01 & 0.105 \\
\hline $0.5 \mathrm{MHz}$ & $1.00^{\mathrm{a}}$ & 0.031 & 1.83 & 0.054 \\
\hline
\end{tabular}

\begin{tabular}{|c|c|c|c|c|}
\hline \multirow[b]{3}{*}{$f_{\text {cutoff }}$} & \multicolumn{4}{|c|}{$\xi_{\mathrm{tot}}=2.2 \times 10^{-2}, \sigma_{\mathrm{BPM}}=50 \sigma_{\mathrm{Noise}}$} \\
\hline & \multicolumn{2}{|c|}{$Q^{\prime}=0$} & \multicolumn{2}{|c|}{$Q^{\prime}=15$} \\
\hline & $g\left[10^{-2}\right]$ & $\dot{\varepsilon}\left[\dot{\varepsilon}_{\text {ref }}\right]$ & $g\left[10^{-2}\right]$ & $\dot{\varepsilon}\left[\dot{\varepsilon}_{\text {ref }}\right]$ \\
\hline Ext. BW & 0.33 & 0.200 & 0.76 & 0.358 \\
\hline $20 \mathrm{MHz}$ & 0.55 & 0.161 & 0.62 & 0.267 \\
\hline $0.5 \mathrm{MHz}$ & 0.37 & 0.147 & 0.39 & 0.245 \\
\hline
\end{tabular}

\begin{tabular}{lccccc}
\hline \hline & \multicolumn{2}{c}{$\xi_{\text {tot }}=2.2 \times 10^{-2}, \sigma_{\text {BPM }}=25 \sigma_{\text {Noise }}$} \\
\cline { 2 - 3 } \cline { 5 - 6 }$f_{\text {cutoff }}$ & \multicolumn{2}{c}{$Q^{\prime}=0$} & & \multicolumn{2}{c}{$Q^{\prime}=15$} \\
\cline { 2 - 3 } \cline { 5 - 6 } & $g\left[10^{-2}\right]$ & $\dot{\varepsilon}\left[\dot{\varepsilon}_{\text {ref }}\right]$ & & $g\left[10^{-2}\right]$ & $\dot{\varepsilon}\left[\dot{\varepsilon}_{\text {ref }}\right]$ \\
\hline Ext. BW & 1.79 & 0.179 & & 1.63 & 0.306 \\
$20 \mathrm{MHz}$ & 1.88 & 0.107 & & 1.72 & 0.194 \\
$0.5 \mathrm{MHz}$ & $1.00^{\mathrm{a}}$ & 0.085 & & 1.25 & 0.161 \\
\hline
\end{tabular}

${ }^{\mathrm{a}}$ Did not consider gains larger than the largest stable gain in simulations.

separately, with a standard deviation of the relative error of $4.3 \%$. The optimal gain is in general smaller for a smaller cutoff frequency. The minimal emittance growth rate at the optimal gain for cutoff frequencies $0.5 \mathrm{MHz}, 20 \mathrm{MHz}$, and Ext. BW are gathered in Table II.

The scan of the average emittance growth rate of 128 bunches has been repeated with $Q^{\prime}=15$, which is presented in Fig. 7. The trends are the same as with $Q^{\prime}=0$, except for a larger emittance growth rate, as was also found in Sec. III A. Especially the emittance growth rate driven by the LF noise alone has increased. Furthermore, some configurations that were unstable have been stabilized with the introduction of the chromaticity. The emittance growth rates in the simulations with both types of noise are equal to the sum of the growth rates found with each type of noise separately with a standard deviation of the relative error of $3.0 \%$. The minimal emittance growth rate and optimal gain for three bandwidths are gathered in Table II. It has been 


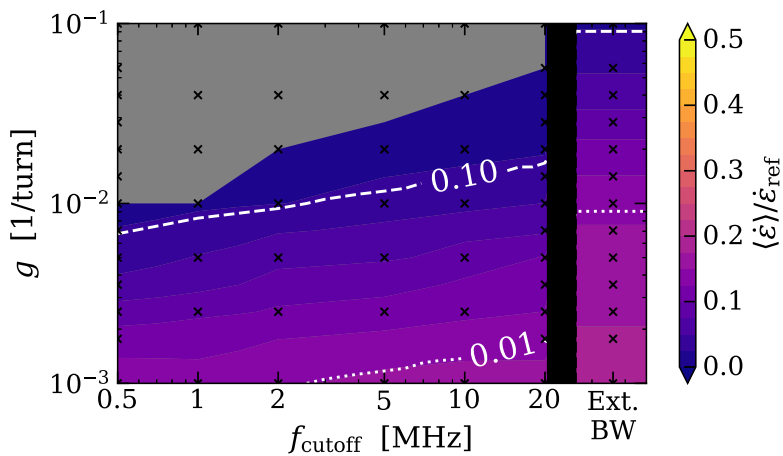

(a)

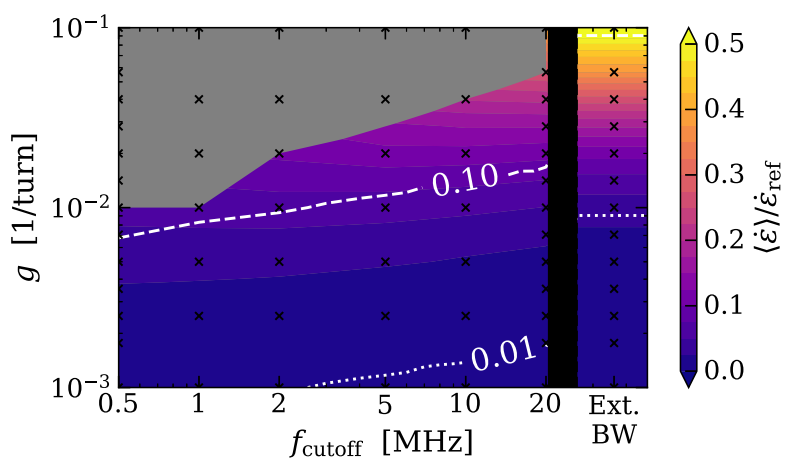

(b)

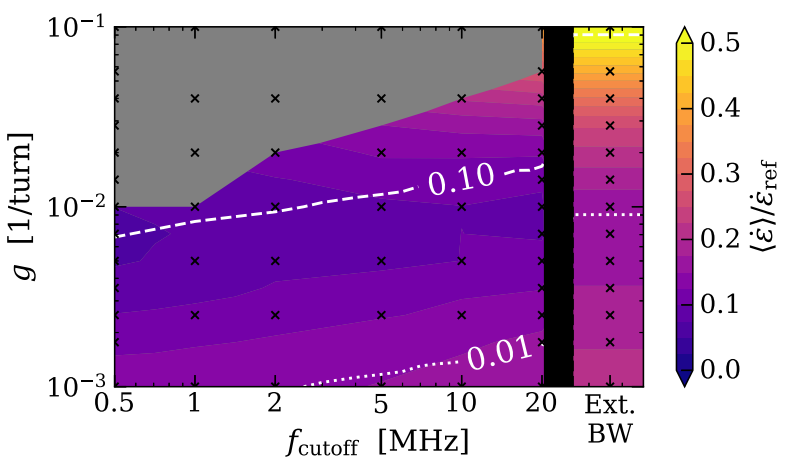

(c)

FIG. 6. Average emittance growth rate for 128 bunches separated by $25 \mathrm{~ns}$, due to LF noise in (a), BPM noise in (b), and both in (c), in a configuration with a beam-beam interaction and $Q^{\prime}=0$. The black crosses mark the simulations. The area marked gray contains configurations $\left(f_{\text {cutoff }}, g\right)$ for which the feedback loop is unstable. The configurations at the edge of the gray area were stable. The white curves are contours of constant maximum multibunch gain $g \tilde{N}$.

tested to operate the LHC with Ext. BW, but it was found that this led to a significantly increased emittance blow-up [23], in agreement with these results. Therefore, the LHC was in run 2 operated with $f_{\text {cutoff }}=20 \mathrm{MHz}$ when in collision.

The transverse feedback system in the LHC is being upgraded during the current long shutdown, in part to reduce the BPM error $[23,24]$. The system will use 4 instead of 2 pickups per plane per beam. The signals from

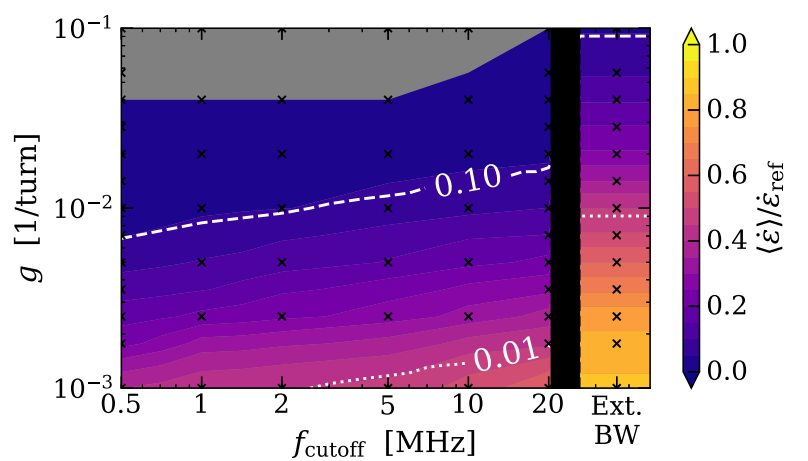

(a)

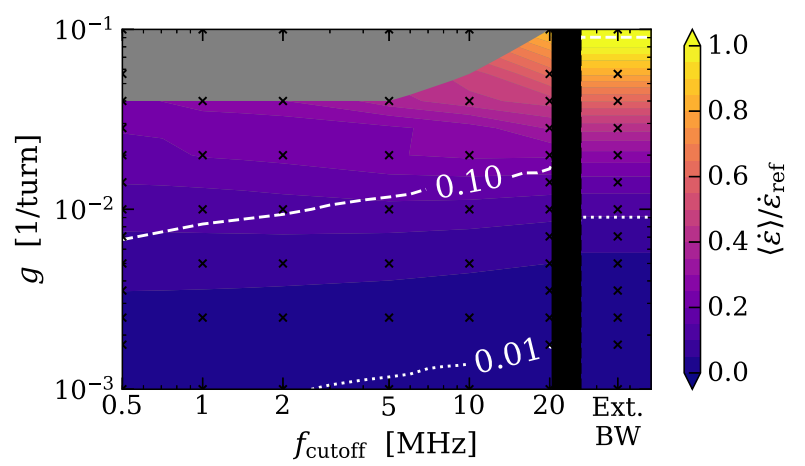

(b)

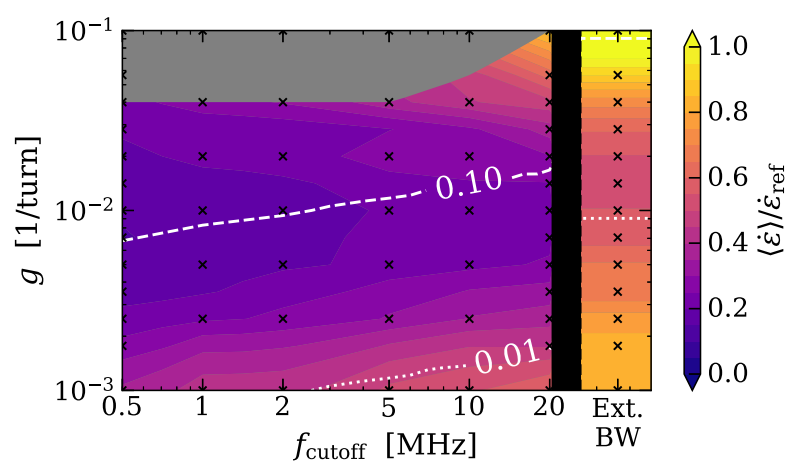

(c)

FIG. 7. Average emittance growth rate for 128 bunches separated by $25 \mathrm{~ns}$, due to LF noise in (a), BPM noise in (b), and both in (c), in a configuration with a beam-beam interaction and $Q^{\prime}=15$. The black crosses mark the simulations. The area marked gray contains configurations $\left(f_{\text {cutoff }}, g\right)$ for which the feedback loop is unstable. The configurations at the edge of the gray area were stable. The white curves are contours of constant maximum multibunch gain $g \tilde{N}$.

the pickups will be treated differently, to reduce effects caused by long $(>700 \mathrm{~m})$ transmission lines between the tunnel and the surface building. The main improvement should come from a new generation radio-frequency frontend, a more advanced sampling scheme, and utilization of additional signal-processing features in the digital domain before the measured beam position is sent to the transverse feedback loops. The goal is to reduce the BPM error by a factor 3 or more. 


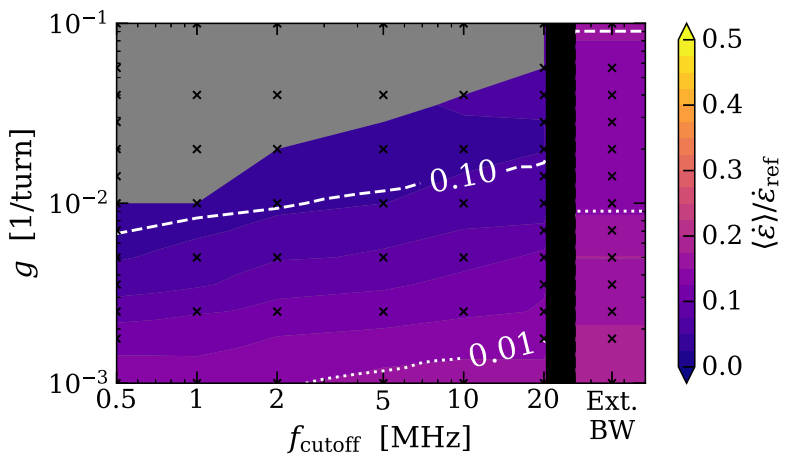

(a)

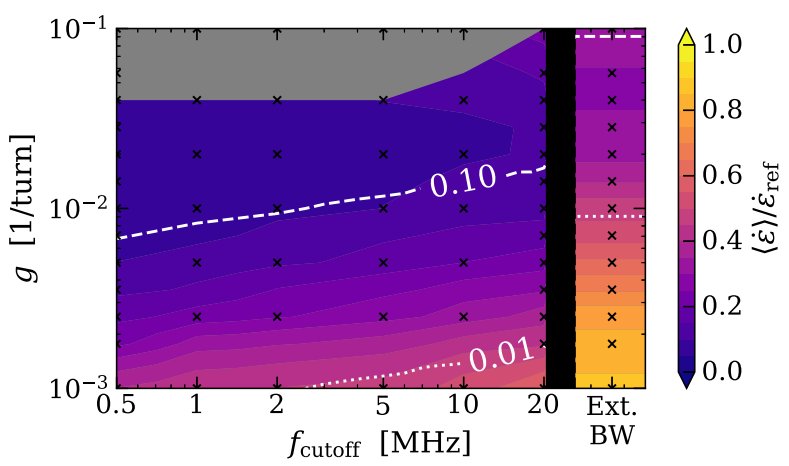

(b)

FIG. 8. Average emittance growth rate for 128 bunches separated by $25 \mathrm{~ns}$, after halving the BPM noise currently in the LHC, in a configuration with a beam-beam interaction and $Q^{\prime}=0$ in (a) and $Q^{\prime}=15$ in (b). The black crosses mark the simulations. The area marked gray contains configurations $\left(f_{\text {cutoff }}, g\right)$ for which the feedback loop is unstable. The configurations at the edge of the gray area were stable. The white curves are contours of constant maximum multibunch gain $g \tilde{N}$.

If one would achieve a halving of the BPM error, the BPM noise driven emittance growth rate would be divided by 4 , leading to the emittance growth rates presented in Fig. 8, with both $Q^{\prime}=0$ and $Q^{\prime}=15$. The values are weighted sums of the corresponding simulations with only one type of noise. The reduction of the BPM noise allows for more optimal configurations with all bandwidths, and a larger single-bunch gain in general, as seen by the minimal emittance growth rates and optimal gains gathered in Table II.

The simulations have been rerun with $\xi_{\text {tot }}=0.022$ and $Q_{s}=0.0021$. This is the total head-on beam-beam parameter in the High Luminosity LHC (HL-LHC). With a larger tune spread, a larger gain is required to reduce the emittance growth rate. The minimal emittance growth rate and optimal gain for $Q^{\prime}=0$ and $Q^{\prime}=15$ are gathered in Table II. With $Q^{\prime}=0$ and the current noise ratio, $\sigma_{\mathrm{BPM}}=50 \sigma_{\text {Noise }}$, the Ext. BW is not able to reduce the emittance growth rate. Note that the prediction without chromaticity by Eq. (4) is $\dot{\varepsilon}=0.175 \dot{\varepsilon}_{\text {ref }}$. With this larger beam-beam parameter, a chromaticity of 15 units increases the emittance growth rate less. For the initial noise ratio as in the LHC, $\sigma_{\mathrm{BPM}}=50 \sigma_{\text {Noise }}$, the emittance growth rate is approximately flat up to $g<0.01$, from where it increases due to the BPM noise. The feedback is barely able to reduce the total emittance growth rate, as was also found experimentally [22].

If the goal of reducing the BPM error to $\sigma_{\mathrm{BPM}}=25 \sigma_{\text {Noise }}$ is achieved in the HL-LHC, the feedback does help, but less than with the smaller beam-beam parameter, as listed in Table II. This is because more noise energy will enter discrete beam-beam modes that do not lead to emittance growth. Assuming the same machine noise as in the LHC, the noise in collision in the HL-LHC is $\sigma_{\text {Noise }}=4.65 \times 10^{-5}$ in units of the beam size [22,25], which causes a reference emittance growth rate with full decoherence of $\dot{\varepsilon}_{\text {ref }} / \varepsilon_{0}=4.4 \% / \mathrm{h}$. Hence, with $Q^{\prime}=15$ in the model simulated here, equivalent to two head-on beam-beam interactions with equal phases between the interaction points (IPs) for the two beams, the optimal Ext. BW feedback, with $g=0.0163$, reduces the emittance growth rate to $1.34 \% / \mathrm{h}$. The optimal LBW feedback, with $g=0.0125$ and $f_{\text {cutoff }}=0.5 \mathrm{MHz}$, manages to reduce it further to $0.70 \% / \mathrm{h}$. With a more realistic model of the machine, featuring in particular long-range interactions and different phases between the IPs for the two beams, the discrete beambeam modes are expected to reach the incoherent spectrum. In these conditions the emittance growth rate can be larger than predicted with the model discussed here by a factor up to 2.5 [22].

\section{CONCLUSION}

Transverse feedback systems are required in modern hadron colliders to maintain beam stability and a small beam emittance, and thereby a high luminosity. One main source of emittance growth is the machine noise. Analytical theories have been derived in the past to calculate the reduction of the noise driven emittance growth rate with an Ext. BW/bunch-by-bunch feedback. In this paper, these theories have been extended to an LBW/multibunch feedback, for which a multibunch gain $g_{b m}$ has been found for each bunch $b$ within each multibunch mode of frequency $f_{m}$. The multibunch gain will in general be larger than the single-bunch gain $g$, and thus further reduce the machine noise driven emittance growth rate, if the cutoff frequency of the feedback is larger than the multibunch mode frequency, $f_{\text {cutoff }}>f_{m}$. The multibunch gain does not fundamentally depend on the feedback cutoff frequency, but on the discrete sum of the response function over the neighboring bunches. Thus, different feedback filters with the same cutoff frequency have different efficiencies $g_{b m} / g$. The extended theories agree quantitatively with heavy macro-particle simulations. It still remains to accurately include a linear chromaticity theoretically, even with the bunch-by-bunch feedback. The predicted emittance growth rates for various feedback systems depend on the noise model used in the calculations and simulations. The power 
spectrum of the machine noise is both expected by theories and measured experimentally to be strongest at low frequencies in general. In the LHC, the noise is currently believed to mainly be below the revolution frequency. Such a noise spectrum will tend to drive multibunch modes of low multibunch mode frequencies, supporting the use of an LBW feedback. However, measurements imply that we do not fully understand the machine noise in the LHC. Therefore, there are several ongoing projects that aim at better describing the possible sources of noise in the machine, both what they are and how much they affect the beams. An update of the noise model will naturally require an update of the predicted emittance growth rates.

In addition to the machine noise, the feedback introduces additional noise on its own, due to BPM errors. The corresponding emittance growth rate has been found to be only weakly dependent on the feedback bandwidth, but increases with the single-bunch gain $g$. When the BPM error is non-negligible, LBW feedbacks are superior in limiting the total emittance growth rate, as one can reduce $f_{\text {cutoff }}$ and $g$ simultaneously, while keeping $g_{b m}$ constant. This has been found both in simulations and in experiments.

The ultimate goal of this paper was to find the optimal configuration of a feedback, which is the value pair $\left(f_{\text {cutoff }}, g\right)$ that minimizes the total emittance growth rate, while keeping the beam stable. The optimum depends on the amplitudes of the noise sources, the feedback type, the source and magnitude of the detuning, and thereby the machine. In the LHC in collision in 2018, both the machine noise and BPM noise were non-negligible. It was found experimentally advantageous to operate with an LBW feedback with $f_{\text {cutoff }}=20 \mathrm{MHz}$, with respect to the Ext. $\mathrm{BW}$, a result which has been reproduced in this paper with a simplified numerical model of the LHC. Yet, the optimal feedback working point would have been an LBW feedback with the lowest possible $f_{\text {cutoff }}=0.5 \mathrm{MHz}$ and $g=0.0075$. This optimum would have achieved a reduction of the emittance growth rate of $72 \%$ compared to the optimal Ext. BW feedback, and a reduction of $34 \%$ compared to the optimum with $f_{\text {cutoff }}=20 \mathrm{MHz}$. However, this working point is close to the feedback stability limit, which must be considered in more detail, both theoretically and experimentally. Thus, this study does not encourage efforts toward reducing the lowest possible cutoff frequency of the LBW feedback in the LHC further below $0.5 \mathrm{MHz}$. The linear chromaticity did not strongly affect the optimal feedback working point. However, it was found that reducing the chromaticity both reduces the emittance growth rate and affects the loop stability. Whether the chromaticity should be changed in operation is first and foremost a question of beam stability, and a possible reduction would have to be investigated further with this in mind. By reducing the BPM error, the emittance growth rate will be more machine noise dominated, and the LHC can be operated more optimally with a larger gain. Without reducing the BPM error, the feedback in the HL-LHC will only be able to marginally reduce the emittance growth rate. The feedback will still be valuable in maintaining beam stability.

\section{ACKNOWLEDGMENTS}

The authors would like to thank: Kevin Li for discussions on the implementation of a realistic transverse feedback system in a tracking code; Sofia Kostoglou for discussions on noise in the LHC; and Elias Métral, Gianluigi Arduini and Rogelio Tomás for constructive feedback on this manuscript. This research was supported in part with computational resources at the CERN HPC cluster.

[1] M. Morrone, M. Martino, R. De Maria, M. Fitterer, and C. Garion, Magnetic frequency response of High-Luminosity Large Hadron Collider beam screens, Phys. Rev. Accel. Beams 22, 013501 (2019).

[2] V. Shiltsev, Review of observations of ground diffusion in space and in time and fractal model of ground motion, Phys. Rev. Accel. Beams 13, 094801 (2010).

[3] S. Kostoglou, Noise effects in the Large Hadron Collider (LHC) and its High-Luminosity upgrade (HL-LHC), Ph.D. thesis, National Technical University of Athens, Athens, Greece, 2020, Report No. CERN-THESIS-2020169.

[4] O. Brüning, P. Collier, P. Lebrun, S. Myers, R. Ostojic, J. Poole, and P. Proudlock, LHC Design report, Vol. 1: The LHC Main ring, CERN, Geneva, Switzerland, Report No. CERN-2004-003-V-1, 2004.

[5] W. Höfle, F. Dubouchet, G. Kotzian, and D. Valuch, Performance of the LHC transverse damper with bunch trains, in Proceedings of the 4th International Particle Accelerator Conference, Shanghai, China, May 2013 (JACoW, Geneva, 2013), paper WEPME043, pp. 3022-3024.

[6] G. Sterbini, A. Blas, and S. Gilardoni, Beam-based performance of the CERN PS transverse feedback, in Proceedings of the 54th ICFA Advanced Beam Dynamics Workshop on High-Intensity, High Brightness and High Power Hadron Beams, East-Lansing, MI, USA, Nov. 2014, paper MOPAB10.

[7] R. J. Steinhagen, LHC Beam Stability and Feedback Control, CERN, Geneva, Switzerland, Rep. No. CERN-Thesis2007-058, 2007, https://cdsweb.cern.ch/record/1054826.

[8] G. Kotzian, Possibilities for transverse feedback phase adjustment by means of digital filters, in Proceedings of the 8th International Particle Accelerator Conference, Copenhagen, Denmark, May 2017 (JACoW, Geneva, 2017), pp. 1924-1927.

[9] J. Komppula, X. Buffat, G. Kotzian, K. Li, and D. Valuch, MD4036: New ADT signal processing for large tune acceptance, CERN, Geneva, Switzerland, Rep. No. CERN-ACC-NOTE-2019-0008, 2019, https://cds .cern.ch/record/2668389.

[10] S. Papadopoulou, F. Antoniou, I. Efthymiopoulos, M. Hostettler, G. Iadarola, N. Karastathis, S. Kostoglou, Y. Papaphilippou, and G. Trad, Monitoring and modelling 
of the LHC emittance and luminosity evolution in 2018, J. Phys. Conf. Ser. 1350, 012011 (2019).

[11] V. A. Lebedev, Emittance growth due to noise and its suppression with the feedback system in large hadron colliders, in AIP Conf. Proc. Accelerator physics at the Superconducting Super Collider, Dallas, Texas, USA (AIP, New York, NY, 1995), pp. 396-423.

[12] J. Gareyte, J. P. Koutchouk, and F. Ruggiero, Landau damping, dynamic aperture and octupoles in LHC, CERN, Geneva, Switzerland, Rep. No. CERN-LHC-PROJECTREPORT-091, 1997.

[13] Y. Alexahin, On the Landau damping and decoherence of transverse dipole oscillations in colliding beams, Part. Accel. 59, 43 (1998); Report No. CERN-SL-96-064-AP, https://ab-abp-rlc.web.cern.ch/ap-literature/alexahin-1996 .pdf.

[14] G. V. Stupakov, V. V. Parkhomchuk, and V. D. Shiltsev, Decoherence of a Gaussian beam due to beam-beam interaction, Rep. No. SSCL-Preprint-495, 1993.

[15] O. Brüning, P. Collier, P. Lebrun, S. Myers, R. Ostojic, J. Poole, and P. Proudlock, LHC Design report, Vol. 1: The LHC main ring, CERN, Geneva, Switzerland, 2004.

[16] M. Plancherel, Contribution à l'étude de la représentation d'une fonction arbitraire par les intégrales définies, Rend. Circ. Mat. Palermo 30, 289 (1910).

[17] W. Herr, M. P. Zorzano, and F. Jones, Hybrid fast multipole method applied to beam-beam collisions in the strongstrong regime, Phys. Rev. Accel. Beams 4, 054402 (2001).

[18] X. Buffat, Transverse beams stability studies at the Large Hadron Collider, Ph.D. thesis, École polytechnique fédérale de Lausanne, Lausanne, Switzerland, 2015,
CERN-THESIS-2014-246, https://doi.org/10.5075/epflthesis-6321.

[19] S. V. Furuseth and X. Buffat, Parallel high-performance multi-beam multi-bunch simulations, Comput. Phys. Commun. 244, 180 (2019).

[20] J. S. Pereira-Cubillo and S. V. Furuseth, Non-white transverse noise in COMBIp, CERN, Geneva, Switzerland, Rep. No. CERN-STUDENTS-Note-2019-116, 2019.

[21] J. Tückmantel, Digital generation of noise-signals with arbitrary constant or time-varying spectra, in Proceedings of the 11th European Particle Accelerator Conference (EPAC08), Genoa, Italy, Jun. 2008 (EPS-AG, Genoa, Italy, 2008), paper TUPC103, pp. 1299-1301.

[22] X. Buffat, W. Herr, T. Pieloni, and D. Valuch, Modeling of the emittance growth due to decoherence in collision at the Large Hadron Collider, Phys. Rev. Accel. Beams 23, 021002 (2020).

[23] F. Dubouchet, W. Höfle, G. Kotzian, and D. Valuch, "What you get"-Transverse damper, in Proceedings of the 4th LHC Operations Evian Workshop, Evian-lesBains, France, Rep. No. CERN-ATS-2013-045, 2012, pp. 73-78.

[24] D. Valuch, W. Höfle, J. Komppula, G. Kotzian, S. Rains, M. Söderén, X. Buffat, ADT upgrades for HL-LHC, presented at the 8th HL-LHC Collaboration Meeting, CERN, Geneva, Switzerland, Oct. 2018, https://indico .cern.ch/event/742082/contributions/3084843/.

[25] E. Métral et al., Update of the HL-LHC operational scenarios for proton operation, CERN, Geneva, Switzerland, Report No. CERN-ACC-NOTE-2018-0002, 2018. 